\title{
Gerontologia Ambiental: panorama de suas contribuições para a atuação do gerontólogo
}

\author{
Environmental Gerontology: overview of its contributions for the actions of gerontologists
}

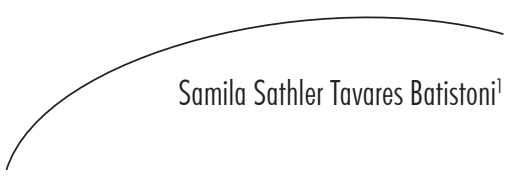

\section{Resumo}

O artigo traz algumas considerações a respeito do campo teórico da Gerontologia Ambiental, ao traçar um breve panorama dos temas clássicos de estudo e aplicação dos novos campos abertos pelas demandas do envelhecimento populacional e políticas públicas decorrentes. $O$ texto busca também salientar o aumento na produção acadêmica internacional no campo, porém não acompanhado na produção nacional. Destaca-se a importância da perspectiva ambiental para as ações práticas do profissional gerontólogo em suas tarefas de gestão de cuidados, serviços e políticas no campo da velhice e dos processos de envelhecimento.

\section{Abstract}

The paper presents some considerations about the theoretical field of Environmental Gerontology, with a brief overview of the classical themes of study and application of the new fields opened up by the demands of population aging and public policy issues arising. The text also seeks to highlight the increase in the international academic field production, but not together in the national production. It highlights the importance of environmental outlook for the practical actions of the professional

Palavras-chave: Gerontologia. Envelhecimento. Perspectiva Ambiental.

Key words: Gerontology. Aging. Environmental Perspective.

Curso de Graduação em Gerontologia, Escola de Artes, Ciências e Humanidades. Universidade de São Paulo. São Paulo, SP, Brasil. 


\section{INTRODUÇÃO}

As perspectivas sobre o envelhecimento humano, sobretudo no que se refere a seus processos comportamentais e adaptativos, foram tradicionalmente pautadas em modelos centrados no declínio biológico e no aumento da idade como variáveis causais e explicativas. Contudo, a partir de meados do século passado, as contribuições metodológicas e conceituais propiciadas pela consideração das variáveis ambientais geraram um notório desenvolvimento do campo da Gerontologia no que diz respeito a teoria, pesquisa e aplicação, contribuindo para uma concepção multidimensional e multidirecional do envelhecimento. ${ }^{1,2}$

Nos últimos quarenta anos, a consideração explícita da influência ambiental sobre o curso e os resultados do envelhecimento propiciou a consolidação de uma subárea denominada "Gerontologia Ambiental", a qual tem oferecido evidências e conceitos importantes para ações de promoção de um envelhecimento saudável e para a construção de sociedades amigas dos idosos. Entre outras definições, a Gerontologia Ambiental é concebida como o campo dedicado a descrição, explicação, modificação ou otimização da relação entre as pessoas idosas e seu entorno socioespacial, e, enquanto campo multidisciplinar, alimenta-se das contribuições advindas das diversas ciências. ${ }^{3,4}$

Embora ainda considerado por alguns como um campo de estudos e não enquanto uma ciência paradigmática, ${ }^{5}$ o desenvolvimento desse campo tem permitido compreender os determinantes contextuais do envelhecimento e sua influência sobre a heterogeneidade na experiência dos idosos. Uma de suas proposições clássicas é que as condições ambientais que permitem aos idosos desempenharem comportamentos biológicos, sociais e psicológicos adaptativos estão entre as dimensões mais relevantes para o envelhecer com qualidade de vida. ${ }^{6}$

Nesse sentido, o presente texto busca trazer algumas considerações a respeito do campo teórico da Gerontologia Ambiental, ao traçar um breve panorama dos temas clássicos de estudo e aplicação dos novos campos abertos pelas demandas do envelhecimento populacional e das políticas públicas decorrentes destas. O texto busca também salientar a importância da perspectiva ambiental para as ações práticas do profissional gerontólogo em suas tarefas de gestão de cuidados, serviços e políticas no campo da velhice e dos processos de envelhecimento.

Gerontologia Ambiental: conceitos clássicos e tendências atuais

Do ponto de vista histórico, a Gerontologia Ambiental é fruto de contribuições teóricas de diversas áreas, como da Sociologia Urbana da Escola de Chicago nos anos 1920, da Psicologia Social de Kurt Lewin na década de 30, das teorias da aprendizagem que dominaram a Psicologia nas décadas de 40 e 50 e do desenvolvimento da Psicologia Ambiental na década de 60.

Uma vez que o termo "ambiente" dentro de cada uma dessas áreas abarca influências sobre o envelhecimento de naturezas diversas, tais como físicas, sociais, organizacionais e culturais, o termo "ambiente sociofísico" tem sido utilizado para especificar seu foco de estudo considerando as transações do indivíduo com o ambiente físico não apenas no que se refere a seus atributos objetivos, mas a partir de sua interpretação social, significados compartilhados e contextualização histórica. Contudo, o campo ainda é caracterizado por um pluralismo de perspectivas, fruto das contribuições interdisciplinares e da consideração dos diferentes níveis de análise teóricos e práticos. ${ }^{4}$

Assim, o espectro de temáticas com as quais a Gerontologia Ambiental está fortemente comprometida atravessa um continuum de análise envolvendo desde o microambiente, representado pelo ambiente doméstico e privado, os arranjos de moradia e a satisfação residencial, passando pelos mesoambientes, tais como os contextos institucionais, até os macroambientes que estruturam a experiência do envelhecimento, tais como o estudo das transações com os contextos urbanos/rurais, questões de vizinhança, segurança, acessibilidade e políticas públicas. 
Embora alguns autores considerem as décadas de 1970 e 1980 como "anos de ouro" na produção das teorias em Gerontologia Ambiental, ${ }^{7}$ vislumbra-se um atual florescimento do campo no panorama internacional, abarcando as contribuições das perspectivas teóricas em Psicologia life-span e Sociologia life-course, da Geografia Social e das intervenções práticas nos campos da Arquitetura, Engenharia e Terapia Ocupacional.

Wahl \& Oswald, ${ }^{7}$ expoentes da Gerontologia Ambiental atual, destacam as três grandes questões e desafios com os quais o campo está envolvido. O primeiro desafio é compreender como os indivíduos (à medida que envelhecem) manejam as oportunidades e restrições nas condições ambientais sociofísicas. O segundo diz respeito à necessidade de clarificar as conexões entre as dimensões objetivas e subjetivas na relação dos indivíduos idosos com o ambiente. $\mathrm{O}$ terceiro desafio é examinar as contribuições das transações pessoa-ambiente para os diferentes cursos (normal, patológico ou bem-sucedido) e respostas (bem-estar, autonomia, identidade, saúde física e mental) em termos de envelhecimento.

Seis principais proposições teóricas desenvolvidas entre as décadas de 1970 e 1980, apesar de criticadas por se pautarem em perspectivas de transações mais representativas dos micro e mesoambientes, ainda são consideradas fontes dos principais conceitos em Gerontologia Ambiental e com potencial contribuição para as macroanálises. A tabela 1 reproduz o resumo das proposições apresentadas na revisão de Wahl, ${ }^{2}$ em ordem cronológica.

Tabela 1. Contribuições teóricas clássicas para a Gerontologia Ambiental em ordem cronológica.

\begin{tabular}{|c|c|c|}
\hline Modelo teórico & Proposição principal & Referência-chave \\
\hline $\begin{array}{l}\text { Modelo pressão- } \\
\text { competência }\end{array}$ & $\begin{array}{l}\text { O comportamento na velhice é uma função } \\
\text { da competência pessoal em interação com a } \\
\text { pressão ambiental }\end{array}$ & Lawton \& Nahemow ${ }^{8}$ \\
\hline $\begin{array}{l}\text { Modelo congruência } \\
\text { pessoa-ambiente }\end{array}$ & $\begin{array}{l}\text { O comportamento é uma função da } \\
\text { congruência entre as características pessoais } \\
\text { (como necessidades) e o que o ambiente } \\
\text { tem a oferecer (capacidade de suprir tais } \\
\text { necessidades) }\end{array}$ & Kahana ${ }^{9}$ \\
\hline Modelo socioecológico & $\begin{array}{l}\text { Ambientes sociais e físicos influenciam o } \\
\text { comportamento individual e vice-versa }\end{array}$ & Moos \& Lemke ${ }^{10}$ \\
\hline $\begin{array}{l}\text { Visão transacional das } \\
\text { pessoas idosas em seus } \\
\text { ambientes }\end{array}$ & $\begin{array}{l}\text { Aspectos físicos, sociais e autobiográficos } \\
\text { do indivíduo como consequências de viver e } \\
\text { envelhecer em um dado lugar }\end{array}$ & Rowles, Oswald, Hunter ${ }^{11}$ \\
\hline Modelo de estresse & $\begin{array}{l}\text { Processos dinâmicos de avaliação, } \\
\text { enfrentamento e reavaliação embasam as } \\
\text { transações pessoa-ambiente }\end{array}$ & Schooler ${ }^{12}$ \\
\hline $\begin{array}{l}\text { Modelo complementar de } \\
\text { congruência }\end{array}$ & $\begin{array}{l}\text { O bem-estar na velhice é influenciado pela } \\
\text { correspondência entre os recursos ambientais } \\
\text { e necessidades pessoais }\end{array}$ & Carp \& Carp ${ }^{13}$ \\
\hline
\end{tabular}


Dentre os conceitos herdados dessas contribuições, destacam-se os de competência, de pressão ambiental e os desdobramentos desses encontrados na obra de Lawton, ${ }^{8}$ que, por volta da década de 1990, expandiu as hipóteses da docilidade e da proatividade ambiental e postulou a respeito das funções básicas do ambiente para o bem-estar na velhice. Uma breve descrição desses conceitos se faz pertinente, uma vez que, juntamente com contribuições atuais, tem auxiliado a construção de novas estruturas teóricas mais abrangentes.

Segundo Lawton \& Nahemow, competência diz respeito à capacidade funcional de um indivíduo refletida em sua saúde biológica, seu funcionamento sensório-perceptual, suas habilidades motoras, cognitivas e forças do ego. Já a pressão ambiental diz das demandas do ambiente sobre o indivíduo, sejam elas físicas, interpessoais ou sociais. A relação entre competência e pressão geraram a hipótese da docilidade ambiental, ou seja, quanto menos competente é umindivíduo, maior é o impacto do ambiente sobre seu comportamento. Complementarmente, Lawton ${ }^{8}$ propôs a hipótese da proatividade ambiental ao sugerir que o indivíduo não é passivo frente às restrições na competência impostas pelas demandas do ambiente, tendendo a alterar seu meio ou a forma de lidar com seu meio para satisfazer suas necessidades e otimizar suas competências.

Em 1989, Lawton ${ }^{8}$ descreveu as três funções básicas dos ambientes sociofísicos para o funcionamento competente e qualidade de vida na velhice, a saber: suas funções de manutenção, de estimulação e de suporte. A função de manutenção destaca a importância da constância e previsibilidade do ambiente, que contribuem para que o indivíduo atribua significados e derive experiências de lar, lugar, identidade, apego e satisfação residencial. A função de estimulação diz respeito aos aspectos do ambiente que estimulam comportamentos adaptativos, como atividades sociais e de lazer ou mesmo aqueles que reduzem ou ampliam problemas comportamentais em idosos com quadros de comprometimentos físicos e/ou cognitivos. Por fim, a função de suporte diz dos aspectos do ambiente que possuem o potencial para compensar competências perdidas ou reduzidas, aspectos também chamados de "próteses ambientais". Entre eles estão os fatores que promovem segurança, orientação e funcionamento independente.

Ao final da década 1990 e início dos anos 2000, teorias psicológicas sobre o construto de crenças de controle baseadas em uma perspectiva lifespan vieram somar-se às contribuições clássicas no exame das transações entre a pessoa que envelhece e o ambiente. As distinções entre controle primário (esforço para exercer o controle direto sobre os eventos e ambiente externo) e controle secundário (ajustes pessoais, como reatribuição causal, para lidar indiretamente com eventos e fatores externos) propostas por Heckenhausen \& Schulz ${ }^{10}$ e as demonstrações de diminuição no senso de controle primário na velhice auxiliam na compreensão de como os indivíduos manejam o ambiente e as próprias crenças subjetivas para lidar com as diminuições nos recursos pessoais.

Partindo do construto de crenças de controle, Oswald e colaboradores ${ }^{11}$ têm proposto o conceito de crenças de controle relacionadas à residência para examinar as percepções que os idosos possuem na explicação de eventos relacionados ao seu microambiente. Nesse sentido, examinase se o idoso percebe as ocorrências como contingentes ao seu próprio comportamento ou como fruto da ação de eventos incontroláveis, tais como por sorte, fatalidade ou por atuação de outros poderosos. O argumento é que as crenças de controle regulam as trocas da pessoa com seu ambiente, tornando-se especialmente importantes na explicação da autonomia e do bem-estar na velhice. Estudos longitudinais têm revelado aumento nas crenças de controle externo como forma de lidar com os declínios em saúde, funcionalidade ou por ação dos estereótipos sobre a velhice. Porém, segundo Oswald e colaboradores ${ }^{11}$ o baixo senso de 
controle primário sobre os eventos que incidem sobre o microambiente relaciona-se com perdas em autonomia e aumento da dependência.

Dos modelos de congruência ${ }^{12}$ e modelos complementares de congruência, ${ }^{13}$ a Gerontologia Ambiental derivou o conceito de ajuste on desajuste pessoa-ambiente. A mensagem inerente ao conceito de ajuste é que o nível de competência em certos domínios é congruente ao nível de pressão ambiental. Assim, incongruência representaria uma condição de risco à competência, ou, por outro lado, uma condição de estímulo de processos de agência humana e de proatividade frente às restrições ambientais.

Como exemplo da ação da incongruência como fator de estímulo, cabe destacar que, por muito tempo, os estudiosos consideravam a realocação residencial como um fator de risco para o bem-estar e funcionalidade dos idosos. Porém, estudos atuais têm demonstrado que a realocação também pode ser fonte de desenvolvimento na velhice, uma vez que promove o surgimento de novos processos adaptativos. ${ }^{14}$

No intuito de integrar as evidências e construtos oriundos sobretudo das contribuições da Psicologia life-span, Wahl e colaboradores ${ }^{2,3,7}$ oferecem uma estrutura conceitual heurística para a compreensão e exame mais refinado das transações pessoa-ambiente na velhice. Nessa estrutura, há o envolvimento das perspectivas clássicas, mas dois construtos centrais fornecem bases úteis para a integração das perspectivas teóricas, a saber: pertencimento e agência.

Conforme a estrutura conceitual ${ }^{7}$ apresentada na figura 1, pertencimento e agência são os dois construtos relacionados ao alcance das duas tarefas desenvolvimentais centrais a serem alcançadas pelos indivíduos à medida que envelhecem, ou seja, as tarefas de manter-se independente o tanto quanto possível (resultando em senso de autonomia) e de manter a integridade do self (senso de identidade).

\begin{tabular}{cc}
\hline $\begin{array}{c}\text { Processos } \\
\text { pessoa-ambiente }\end{array}$ & $\begin{array}{c}\text { Respostas } \\
\text { desenvolvimentais }\end{array}$ \\
\hline
\end{tabular}

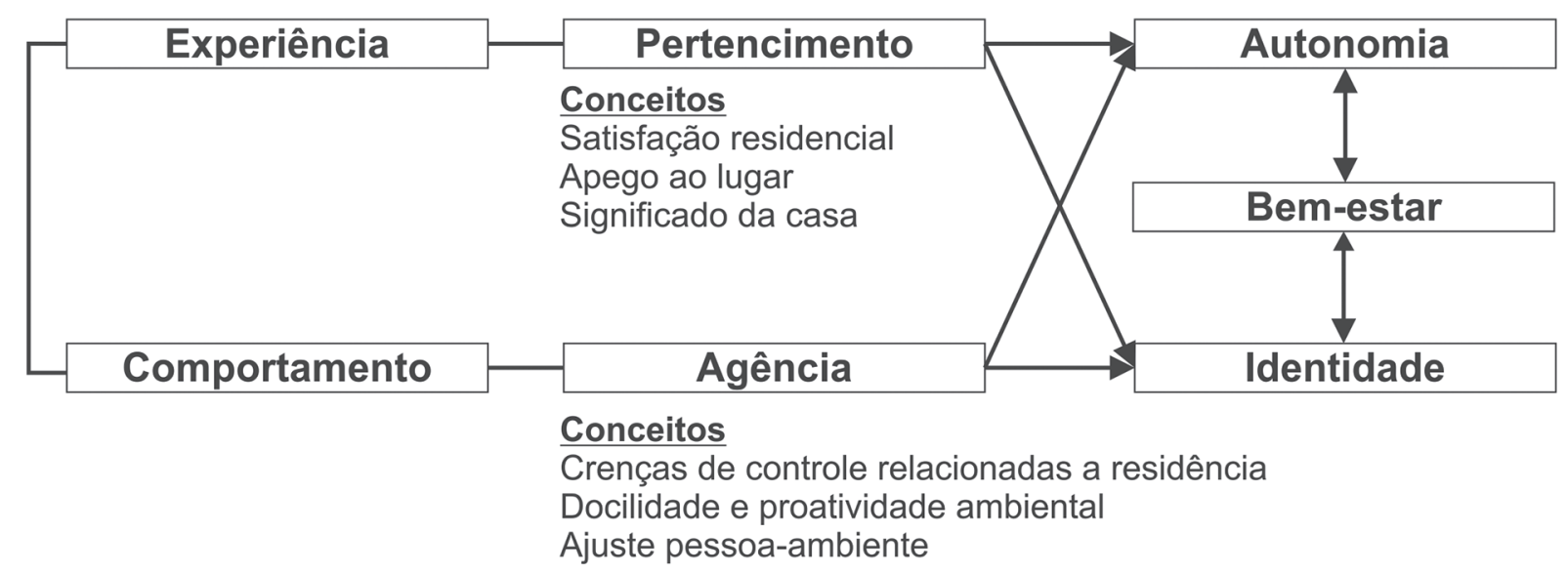

Figura 1. Estrutura conceitual sobre as relações pessoa-ambiente na velhice. ${ }^{7}$ 
Pertencimento diz respeito ao campo da experiência no ajuste pessoa-ambiente, envolvendo as avaliações cognitivas, emocionais e as representações mentais dos ambientes sociofísicos. $\mathrm{Na}$ literatura de pesquisa, o pertencimento tem sido medido por seus correlatos, como satisfação residencial, apego ao lugar e significados atribuídos ao lugar e/ ou compartilhados, e reflete uma orientação ambiental em termos de passado, presente e futuro, reminiscências verbalizadas e planos futuros.

Por outro lado, agência se refere ao campo dos comportamentos, cognições ou práticas sociais orientados a uma meta. É o exercício do controle sobre o ambiente e o manejo das demandas e pressões ambientais ("docilidade") resultando ou não em proatividade (uso ativo do espaço, compensação adaptação, criação de novos espaços) e ajuste.

Wahl \& Oswald $^{7}$ defendem que, por muito tempo, a Gerontologia Ambiental considerou os construtos de pertencimento e agência como processos separados. Entretanto, a estrutura conceitual apresentada assume a considerável interação entre pertencimento, agência e as mudanças desenvolvimentais. Processos comportamentais, cognitivos e emocionais estão envolvidos em processos adaptativos ao longo da vida e principalmente frente às perdas em competência na velhice. Wahl \& Oswald ${ }^{7}$ exemplificam essa interação ao apontar os processos adaptativos de idosos com perdas severas em competência. Segundo os autores, os idosos reduzem objetivamente suas esferas ambientais de ação ao mesmo tempo em que tendem, cognitivamente, a atribuir a essa esfera mais valor, sentido e utilidade do que àqueles ambientes que não lhe são mais acessíveis. Assim, a adaptação na velhice não se refere apenas a comportamentos ou a experiência, mas a ambos os processos em interação. ${ }^{17,18}$

Os resultados da interação entre pertencimento e agência são representados pelas respostas desenvolvimentais de autonomia e identidade. Esses construtos relacionam-se fortemente com o bem-estar na velhice, termo que, tomado globalmente, refere-se a avaliações afetivas e cognitivas positivas no que tange à própria vida e às repostas positivas em saúde física e mental. ${ }^{19}$

A estrutura conceitual apresentada tem auxiliado a Gerontologia Ambiental em seus desafios centrais, a saber, nas tarefas de descrição sobre como os idosos manejam as oportunidades e restrições nas condições ambientais sociofísicas, no exame das conexões entre as dimensões objetivas e subjetivas na relação dos indivíduos idosos com o ambiente e na explicação das contribuições das transações pessoa-ambiente para os diferentes cursos e respostas em termos de envelhecimento.

Entretanto, outras contribuições atuais ao campo da Gerontologia Ambiental também podem ser destacadas, cabendo ressaltar aquelas advindas das perspectivas sociais de inspiração lifecourse. Estudiosos no campo da Geografia Social, como Goland, ${ }^{20}$ têm buscado compreender, a partir de metodologias qualitativas e a partir da perspectiva dos próprios idosos, as dimensões intencionais e ativas destes no uso, manipulação e desempenho de tarefas em seus ambientes sociofísicos.

Goland ${ }^{20}$ defende a inclusão da perspectiva de tempo na pesquisa em Gerontologia Ambiental, uma vez que as experiências dos idosos em seus ambientes correntes são influenciadas pelo contexto e significado histórico construído na relação com o ambiente, além das antecipações futuras que tecem a partir dessas relações. O referido autor considera que a inclusão da dimensão de tempo pode ser metodologicamente auxiliada pelas abordagens de "história de vida" advindas da Psicologia e Antropologia, das análises das "carreiras" residenciais descritas pelos demógrafos e pelos estudos longitudinais no campo da Geriatria a respeito das trajetórias de processos patológicos e de incapacidade.

Investimentos de pesquisa em níveis comunitários e populacionais de análise também compõem o panorama atual do 
campo, como as contribuições dos estudos dos impactos socioespaciais do envelhecimento populacional. Dados provenientes da Geografia do Envelhecimento, como os de SánchezGonzález, ${ }^{21}$ por exemplo, têm lançado luz sobre esses impactos no contexto mexicano e latinoamericano e incentivado o aprofundamento em temáticas como as do ambiente construído e a qualidade de vida de idosos, a das estratégias residenciais para manutenção dos idosos nas comunidades, da vulnerabilidade climática e ambiental a que idosos podem estar expostos, e ainda sobre o apego ao lugar, exclusão social, lazer, turismo e planejamento gerontológico.

Outro conjunto de contribuições atuais à Gerontologia Ambiental advém dos estudos de intervenção desenvolvidos nos campos aplicados da Arquitetura, Engenharia, Fisioterapia e Terapia Ocupacional. Kendig ${ }^{4}$ destaca que, embora classicamente a Arquitetura, a Engenharia e os urbanistas tenham contribuído com o saber em Gerontologia Ambiental, destacam-se atualmente os estudos de intervenção desenvolvidos por profissionais da saúde. Entre estes últimos, tem havido o amplo reconhecimento, no contexto da prevenção e promoção da saúde, da importância do conceito "aging in place", que em linhas gerais sintetiza as vantagens que o idoso possui ao envelhecer no seu contexto residencial, desfrutando de sua vida comunitária. ${ }^{22}$ Exemplos de temas frequentes nesses estudos são as questões de acessibilidade, segurança e prevenção de quedas que, além de contribuírem no trabalho de intervenção clínica e aplicações de "autoajuda" a idosos e familiares, retroalimentam a teoria.

Estudos sobre acessibilidade têm revelado uma importante troca entre a teoria e a prática. Uma vez que a Gerontologia Ambiental postula a interação entre aspectos objetivos e subjetivos da relação pessoa-ambiente, a acessibilidade tem sido considerada não como o número objetivo de barreiras ambientais impostas versus o grau de preservação da capacidade, mas como a percepção do ajuste individual frente às barreiras ambientais. A percepção de ajuste ou desajuste é fruto da interação entre critérios objetivos e subjetivos do indivíduo. Iwarsson ${ }^{23}$ encontrou, por exemplo, que a acessibilidade, assim concebida, foi mais fortemente relacionada à capacidade funcional de idosos do que o número objetivo de barreiras ambientais existentes.

Estudos de intervenção também fornecem à Gerontologia Ambiental elementos importantes para a análise de temáticas como a da incidência de quedas entre idosos. Em estudo de metaanálise, Oswald e colaboradores ${ }^{11}$ encontraram relações significativas entre intervenções no ambiente doméstico e respostas de diminuição de incapacidades. Contudo, encontraram relações mais fracas entre intervenções ambientais (como única frente de intervenção) e incidência de quedas. Tais resultados demonstram, por exemplo, a necessidade de abordagens multidimensionais na compreensão teórica e na intervenção com idosos com histórico de quedas.

Observa-se, portanto, que o status atual da Gerontologia Ambiental é plural em termos de níveis de análise do seu objeto de estudo, o que enriquece, mas desafia o campo na construção de sua perspectiva teórica. Semelhantemente ao quadro teórico da Gerontologia como um todo, o campo tem sido constituído por microteorias explicativas que buscam abarcar sua influência multidisciplinar.

No Brasil, ainda existem poucos estudos na área. A produção existente reflete, em geral, estudos relativos a idosos e envelhecimento provenientes da Psicologia Ambiental ${ }^{24-26}$ ou estudos relativos a intervenções ambientais com idosos com comprometimentos físicos ou cognitivos, ${ }^{27}$ de levantamento, e análise de fatores relacionados a ocorrência e prevenção de quedas $^{28}$ ou, ainda, compondo a análise dos determinantes ambientais da promoção de saúde. ${ }^{29}$ Salienta-se que o campo possui grande potencial de estudos e construção de teorias pautadas na coleta e análise de dados contextualizados sobre as relações entre a pessoa que envelhece e seu ambiente. Novas demandas provenientes do cenário demográfico brasileiro e mundial fornecem também estímulos ao campo. 


\section{Novas demandas à Gerontologia Ambiental}

A urbanização, a globalização e o envelhecimento populacional têm sido considerados as principais forças a moldar as sociedades no século XXI. Assim sendo, as concepções mais atuais em Gerontologia e as preocupações públicas decorrentes dessas forças têm trazido demandas teóricas e práticas sob uma perspectiva macrossocial à Gerontologia Ambiental.

Alguns reflexos dessas demandas podem ser encontrados em conceitos e iniciativas públicas, tais como as denominadas "sociedades e/ou cidades amigas dos idosos". Segundo o Guia Global da Iniciativa "Cidade amiga do idoso", desenvolvido pela Organização Mundial da Saúde, ${ }^{30}$ uma "cidade-amiga" é aquela que estimula o envelhecimento ativo ao otimizar oportunidades para a saúde, participação e segurança, para aumentar a qualidade de vida à medida que as pessoas envelhecem. Segundo o mesmo Guia, em termos práticos, uma "cidadeamiga" adapta suas estruturas e serviços para que estes sejam acessíveis aos idosos e promovam a inclusão daqueles com diferentes necessidades e graus de capacidade.

O direcionamento da Gerontologia Ambiental para as prioridades públicas e sociais é extremamente relevante frente à necessidade de compreender e promover o uso proativo dos ambientes por idosos que desejam se manter saudáveis e em suas próprias casas e comunidades. Esse direcionamento difere significativamente da ênfase de pesquisa de trinta anos atrás, em que a atenção (pública, social e de pesquisa) se centrava na minoria frágil dos idosos, residente em contextos institucionais. ${ }^{3}$

Torna-se premente a teorização nos níveis micro e mesoambientais em conjunto com as macroperspectivas. A consideração das tensões e pressões ambientais exercidas pelo contexto urbano sobre os processos de envelhecimento pode oferecer luz à compreensão dos processos de pertencimento e agência e das respostas em termos de bem-estar dos idosos na sua relação com os ambientes.

Um dos temas sobre as relações entre os níveis micro, meso e macroambientais envolve a questão da exclusão social de idosos. Observa-se, por exemplo, nas coortes idosas, que o desejo de residir nas cidades por razões de acesso a recursos de saúde, lazer, cultura e participação social tem sido minado pela conjunção "urbanização, políticas econômicas, globalização e novas tecnologias". Assim, as cidades geram barreiras intergeracionais, refletindo-se, no nível macro, em desigualdades em qualidade de vida e no acesso a recursos e serviços. Reflete-se também no nível mesoambiental pela formação de "guetos" de participação e trocas interpessoais, e no nível microambiental, em resultados negativos em termos de bem-estar físico e psicológico.

Stahl \& Iwarsson $^{31}$ consideram que, para a construção de uma cultura de ajuste pessoaambiente nas sociedades em envelhecimento, é crítico que a Gerontologia Ambiental contribua considerando, em sua teoria e prática, a importância da organização dos espaços públicos na redução de "áreas de ansiedade" e na promoção de segurança e participação por meio de designs livres de barreiras arquitetônicas e geracionais.

Cabe salientar, contudo, que no plano metodológico, a ampliação do escopo da Gerontologia Ambiental considerando as macroperspectivas implica desafios frente a grande heterogeneidade e variabilidade dos contextos de envelhecimento e do amplo espectro de condições de saúde, competência e bem-estar dos idosos. Gitlin, ${ }^{32}$ por exemplo, propõe a aliança entre as metodologias qualitativas e quantitativas na compreensão dos diversos significados da relação entre os processos de envelhecimento e os contextos urbanos.

No âmbito da intervenção (clínica ou em políticas públicas), o emprego dessa combinação de metodologias é estimulado pelo Guia Cidade Amiga dos Idosos da OMS, ${ }^{30}$ o qual preconiza a formação de grupos focais com idosos e com a comunidade 
para conhecer as percepções dos indivíduos e suas relações com as estruturas das cidades, das atitudes sociais frente ao envelhecimento, das formas de comunicação e fornecimento de serviços a idosos. A recomendação é que as intervenções e projetos de adequação dos espaços públicos e privados para idosos sejam precedidos por embasamento oferecido pela pesquisa quali-quantitativa com os atores sociais. Investimentos recentes de pesquisa e intervenção na França, ${ }^{33}$ Canadá $^{34}$ e Brasil,${ }^{35}$ sob a ótica das iniciativas "cidades amigas dos idosos", têm caminhado nessa direção. Estudos realizados na cidade de São Paulo-SP, por exemplo, têm derivado indicadores de natureza objetiva e subjetiva relevantes para as políticas públicas e contextualização de práticas gerontológicas. ${ }^{34}$

Considerar as macroperspectivas em Gerontologia Ambientalimplica também conceber as relações entre envelhecimento e ambiente como extremamente dinâmicas no que tange às dimensões de mudanças históricas, culturais, ecológicas, de arranjos sociais e familiares e de inovações tecnológicas. De forma resumida, Wahl \& Oswald ${ }^{7}$ destacam, além dos desafios impostos na arena societal e política (como a urbanização e novas tecnologias), outros desafios atuais ao campo - entre eles, a compreensão das relações dos idosos com ambientes residenciais multigeracionais, com os novos contextos e modelos de cuidados em saúde e novos estilos de vida das coortes de idosos atuais. Pode-se também acrescentar a esses, a necessidade de investimento na compreensão das implicações das mudanças climáticas e do impacto das catástrofes naturais sobre o curso do envelhecimento e nas respostas em termos de adaptação, tema ainda pouco explorado na literatura.

\section{Contribuições à atuação do profissional gerontólogo}

O campo da Gerontologia Ambiental possui grande valor no fornecimento de bases teóricas e práticas para o trabalho do profissional em Gerontologia. Frente às novas demandas populacionais, o gerontólogo é o novo profissional que surge no cenário da gestão de serviços (clínicos, recreativos, institucionais) e políticas, estando habilitado a atuar e produzir saberes relacionados ao bem-estar das pessoas idosas, das famílias e da sociedade em envelhecimento. Nesse sentido, a Gerontologia Ambiental convoca o gerontólogo a atentar para o plano das influências dos ambientes sociofísicos em suas tarefas de avaliação e intervenção. $O$ planejamento ambiental deve ser considerado um componente necessariamente presente nos processos de micro e macrogestão.

No que se refere à atuação do gerontólogo no campo clínico, no apoio e aconselhamento de famílias e grupos, o exame das relações indivíduo-ambiente oferece caminhos para a avaliação e identificação de barreiras à autonomia, à segurança, à independência e ao bem-estar. Tal exame deve levar à consideração de que os idosos possuem diferentes esferas de competência nas quais a proatividade ambiental pode ser exercida frente às pressões ambientais e que a tomada de decisões na gestão deve otimizar a congruência entre as necessidades e as competências.

A Gerontologia Ambiental leva o gerontólogo também a considerar que a relação com o ambiente interfere no bem-estar subjetivo e regulação dos estados afetivos dos idosos, uma vez que o envelhecimento expõe cada vez mais os indivíduos às influências das condições de docilidade ambiental. Contudo, ao mesmo tempo, chama a atenção para que as iniciativas em microgestão, mesmo em casos de maior fragilização ou comprometimentos, não deixe de incluir o idoso nas decisões, potencializando ao máximo seu senso de autonomia e agência.

Nas tarefas de gestão de serviços e desenvolvimento de projetos, programas e políticas, o gerontólogo pode usufruir das contribuições teóricas e de intervenção desse campo, os quais têm apontado as relações entre características objetivas e subjetivas do design ambiental e o bem-estar das pessoas idosas. Intervenções no âmbito da acessibilidade nos equipamentos sociais e de saúde, da eliminação 
de barreiras geracionais na comunidade e na criação de espaços de promoção de convivência social e produtividade do idoso devem visar à otimização das funções de manutenção, estimulação e suporte ambiental.

Por fim, a atuação do gerontólogo no âmbito da construção de políticas públicas em saúde, habitação, trabalho, previdência e lazer envolve intrinsecamente a consideração dos determinantes ambientais da qualidade de vida dos idosos. Portanto, a formação do gerontólogo deve necessariamente instrumentalizá-lo para analisar, interpretar e construir propostas de intervenção baseadas nos saberes da Gerontologia Ambiental.

\section{REFERÊNCIAS}

1. Izal M, Fernández-Ballesteros R. Modelos ambientales sobre la vejez. An Psicol 1990;6(2):181-98.

2. Wahl HW. Environmental influences on aging and behavior. In: Birren JE, Schaie KW, editors. Handbook of the psychology of aging. San Diego: Academic Press; 2001. p. 215-37.

3. Wahl HW, Weisman GD. Environmental gerontology at the beginning of new millennium: reflections on its historical, empirical, and theoretical development. Gerontologist 2003;43(5):612-27.

4. Kendig H. Directions in environmental gerontology: a multidisciplinary field. Gerontologist 2003;43(5):611-15

5. Schwarz B. Enviromental Gerontology: what Now? In: Scheidt R, Schwarz B, editors. Enviromental Gerontology: what Now? London, UK: Routledge; 2012. p. 7-22.

6. Lawton MP. Environment and other determinants of well-being in older people. Gerontologist 1983;23(4):349-57.

7. Wahl HW, Oswald F. Environmental perspectives on aging. In: Dannefer D, Phillipson C, editors. International Handbook of Social Gerontology. London: Sage; 2010. p.111-24.

8. Lawton MP. Environmental Proactivity in Older People. In: Bengtson VL, Schaie KW, editors. The Course of Later Life. New York: Springer Publisher; 1989. p. 15-23.

\section{CONSIDERAÇÕES FINAIS}

A relevância das contribuições da Gerontologia Ambiental para as novas demandas do envelhecimento populacional e para as práticas do gerontólogo tem levado a um aumento na produção acadêmica internacional no campo que, no entanto, não tem sido acompanhado na produção nacional.

A necessidade de construir um saber contextualizado deve estimular iniciativas de desenvolvimento de núcleos acadêmicos de estudo sobre a temática, como também a abertura de espaços de discussão e reflexão entre os profissionais envolvidos em Gerontologia.

9. Lawton MP, Nahemow L. Ecology and the aging process. In: Eisdorder C, Lawton MP, editors. Psychology of adult development and aging. Washington: American Psychological Association; 1973. p. 657-68.

10. Heckhausen J, Schulz R. A life-span theory of control. Psychol Rev 1995;102(2):284-304.

11. Oswald F, Wahl HW, Schilling O, Iwarsson S. Housing-related control beliefs and independence in activities of daily living in very old age. Scand J Occup Ther 2007;14(1):33-43.

12. Kahana E. A congruence model of personenvironment interaction. In: Lawton MP, Windley PG, Byerts TO, editors. Aging and the environment: teoretical approaches. New York: Springer; 1982. p. 97-121.

13. Carp FM, Carp A. A complementary/congruence model of well-being or mental health for the community elderly. In: Altman I, Lawton MP, Wohlwill JF, editors. Human behavior and environment: elderly people and the environment. New York: Plenum Press; 1984. p. 279-336.

14. Rowles GD, Oswald F, Hunter EG. Interior living environments in old age. Annu Rev Gerontol Geriatr 2004;22:167-93.

15. Moos RH, Lemke S. Specialized living environments for older people. In: J Birren JE, Schaie KW, editors. Handbook of the Psychology of Aging. New York: Van Nostrand Reinhold; 1985. p. 864-89. 
16. Schooler KK. Response of the elderly to environment a stress-theoretical perspective. In: Lawton MP, Windley PG, Byerts TO, editors. Aging and the environment: theoretical approaches. New York: Springer; 1982. p.103-24.

17. Lawton MP. Residential environment and selfdirectedness among older people. Am Psychol 1990;45(5):638-40.

18. Oswald F, Rowles GD. Beyond the relocation trauma in old age: new trends in today's elders' residential decisions. In: Wahl HW, Tesch-Römer C, Hoff A, editors. New Dynamics in Old Age: environmental and societal perspectives. Amityville, New York: Baywood Publisher; 2006. p. 127-52.

19. Ryff CD. Beyond Ponce de Leon and life satisfaction: new directions in quest of successful ageing. Int J Behav Dev 1989;12(1):35-55.

20. Golant S. Conceptualizing time and behavior in environmental gerontology: a pair of old issues deserving new thought. Gerontologist 2003;43(5):638-48.

21. Sánchez-González D. Urban demographic aging and its socio-spatial repercussions in México: challenges for gerontology planning. Rev Geogr Norte Gd 2007;(38):45-61.

22. Chapin R, Dobbs-Kepper D. Aging in place in assisted living: philosophy versus policy. Gerontologist 2001;41(1):43-50.

23. Iwarsson S. A long-term perspective on $\mathrm{p}-\mathrm{e}$ fit and ADL dependence among older Swedish adults. Gerontologist 2005;45(3):327-36.

24. Capone V. Satisfação de idosos em ambientes de vizinhança de duas regiões do Distrito Federal [dissertação]. Brasília: Universidade de Brasília; 2001.

25. Khoury HTT. Controle primário e controle secundário: relação com indicadores de envelhecimento bem-sucedido [dissertação]. Brasília: Universidade de Brasília; 2005.
26. Cupertino AP. Avaliação pós-ocupação de instituições de idosos no Distrito Federal: uma aplicação do modelo de Moos e Lemke [dissertação]. Brasília: Universidade de Brasília; 1996.

27. Pascale CC. Ergonomia e Alzheimer: a contribuição dos fatores ambientais como recurso terapêutico nos cuidados de idosos portadores da demência do tipo Alzheimer [dissertação]. Florianópolis: Universidade Federal de Santa Catarina; 2002.

28. Messias M, Neves R. A influência de fatores comportamentais e ambientais domésticos nas quedas em idosos. Rev Bras Geriatr Gerontol 2009;12(2):275-82.

29. Cassol PB. A Gerontologia interface o meio ambiente como estratégia no cuidado e promoção da saúde. Reget 2012;6(6):1043-48.

30. Organização Mundial da Saúde. Guia Global. Cidade amiga dos idosos. Genebra: OMS; 2008.

31. Ståhl A, Iwarsson S. Let's go for a walk : a project focusing on accessibility, safety and security for older people in the outdoor environment. Gerontologist 2004;44(Spec no I):151-51.

32. Gitlin L. Conducting research on home environments: lessons learned and new directions. Gerontologist 2003;43(5):628-37.

33. Pennec S, Le Borgne-Uguen F. Quimper: ville amie des aînés - OMS, Diagnostic realize auprès des habitants et des professionnels. Brest, France: ARSUBO; 2012.

34. Garon S, Beaulieu M, Paris M, Veil A, Laliberté A. The implementation of "Cities Friends of the Elderly in Québec": the importance of the participation of all actors. Kairós 2012;15(6):157-75.

35. Graeff B, Domingues MA, Betesti MLT. Bairro amigo do idoso no Brás: percepções sobre os migrantes internacionais. Kairós 2012;15(6):177-69. 